\title{
Dissolution Testing of Herbal Medicines: Challenges and Regulatory Standards in Europe, the United States, Canada, and Asia
}

\author{
Lucia Disch ${ }^{1,2, *}$, Jürgen Drewe ${ }^{1}$, and Gert Fricker ${ }^{2}$ \\ ${ }^{1}$ Max Zeller Söhne AG, Romanshorn, Switzerland \\ ${ }^{2}$ Institute of Pharmaceutics and Biopharmacy, Ruprecht-Karls University, Heidelberg, Germany
}

\begin{abstract}
Herbal medicines are the oldest method for treating diseases and at the same time still the most commonly used worldwide. Although people in developing countries depend on traditional herbal medicines, herbal products also play an important role in the healthcare systems of industrialized countries due to ongoing health trends for substituting natural products for potentially harmful chemicals. The regulatory standards of various authorities for herbal medicines are not harmonized in regional classification and quality requirements. It is particularly difficult to oversee authority requirements for dissolution testing of herbal medicines due to widely varying regulations. The aim of this article is to give a short overview of regulatory classification and dissolution standards of herbal medicinal products with regard to regional differences in Europe, the United States, and Asia. Furthermore, challenges in dissolution method development for herbal medicines are discussed. Because the ingredients of herbal medicinal products often cover a mixture of multiple herbal constituents, dissolution method development is much more complex than for defined single constituents.
\end{abstract}

KEYWORDS: Dissolution; dissolution method development; herbal drug; herbal medicine; regulation; Europe; United States; Canada; China; Japan; South Korea.

\section{INTRODUCTION}

$\mathrm{t}$ is estimated that $80 \%$ of the world population relies on herbal medicines (1). Their usage is widespread over all regions and all social classes. People in developing countries in particular depend on traditional herbal medicines, but in industrialized countries there is a growing wish to substitute natural products for potentially harmful chemicals. Thus, a reliable quality control system to ensure the efficacy and safety of herbal medicinal products is essential. The standards of various authorities for herbal medicines are inconsistent in regional regulatory classification and quality requirements. In particular, regulations for dissolution testing of herbal medicines vary considerably, making it difficult to oversee. Pharmacopeial monographs defining dissolution testing standards for chemical solid dosage forms are available (e.g., USP General Chapter $<711>, E P$ 2.9.3, and $J P$ 6.10). The European Medicines Agency (EMA) issued an ICH guideline Q4B annex 7 (R2) on dissolution testing in 2010 (2). In addition, the International Pharmaceutical Federation (FIP) provides a worldwide and independent platform for scientific discussion. In 2003, a working group of FIP outlined suggestions for dissolution testing

*Corresponding author.
(3). Exceptions and special regulations for herbal products are covered in individual monographs such as USP General Chapter $<2040\rangle$. The aim of this paper is to give a short overview about regulatory classification and dissolution standards for herbal medicinal products in Europe, the United States, Canada, and Asia. Furthermore, challenges in dissolution method development for herbal drugs are discussed.

\section{DISSOLUTION IN THE \\ PHARMACEUTICAL INDUSTRY}

Dissolution testing has its beginnings in 1897 when Noyes and Whitney published the first dissolution studies (4). Dissolution is defined as the rate at which a drug substance is dissolved from a solid pharmaceutical dosage form into a liquid medium as a function of time. The correlation between in vitro solution rates of an orally administered drug substance and its plasma concentration was described for the first time in 1957 by Nelson (5). Since then, in vitro dissolution testing has evolved to be a key element in pharmaceutical development and quality control. In vitro dissolution testing now follows regulatory guidelines described in pharmacopeias worldwide. Because dissolution characteristics depend on several 
parameters, monographs define standards for dissolution media, dissolution apparatus, rotation speed, as well as sampling points and temperature depending on the dosage form.

In vitro dissolution testing has become a main surrogate method in pharmaceutical development and serves as the basis for an in vitro-in vivo study. For classification according to the Biopharmaceutics Classification System (BCS), solubility, permeability, and in the case of solid dosage forms, dissolution characteristics are taken into account $(6,7)$. The EMA issued a document on special considerations for the biopharmaceutical characterization of herbal medicinal products (8).

Especially in the case of modified-release dosage forms with predefined drug release profiles, dissolution testing is an indispensable tool. For example, in the development of prolonged-release formulations, the dissolution process has a fundamental importance because this step is ratedetermining in the absorption process. Furthermore, dissolution testing is an excellent prediction parameter in determining the stability of an oral dosage form.

In quality control, dissolution is usually mandatory for release and stability testing of oral formulations. Dissolution results support batch uniformity and batchto-batch consistency as well.

\section{CHALLENGES OF DISSOLUTION METHOD DEVELOPMENT FOR HERBAL DRUGS}

For chemical drugs, dissolution testing is a firmly established tool in pharmaceutical development and quality control. Development and validation of dissolution methods with a focus on oral solid dosage forms is supported by USP General Chapter <1092>. A revision in 2015 achieved a more scientific approach, and the first part of this chapter is termed "Preliminary Assessment" with standards for performing filter compatibility, determining solubility, and selecting media buffers and volumes as well as apparatus type. The second part of the chapter, on method development, focuses on dissolution performance parameters such as deaeration, use of sinkers, agitation, study design, and data handling. Analytical quantification of dissolution samples is covered under Analytical Finish. Other parts of General Chapter <1092> include Automation, Validation, Acceptance Criteria, and References.

For herbal drugs, additional considerations are necessary in the development of dissolution methods. Because the active constituents of herbal medicinal products contain a mixture of multiple herbal ingredients, development of dissolution methods is much more complex than for single actives.

\section{Analytical Marker Substances}

According to the WHO definition $(9,10)$, herbal medicines comprise herbs, herbal materials, herbal preparations, and finished herbal products. Active constituents are defined as parts of plants, plant materials, or herbal preparations such as extracts as well as combinations thereof. Thus, herbal drugs consist of various compounds, not all of which are known in many cases. To obtain a dissolution profile, analytical measurement of a chemically defined substance is required. Therefore, prior to dissolution method development of an herbal drug, one or more analytical marker substances that are characteristic of the appropriate herbal drug and a suitable surrogate for pharmacological activity have to be determined. Constituents that show or contribute to pharmacological activity are selected based on knowledge and commercial availability. Criteria for selecting herbal markers are detailed in a paper from the EMA (11).

Several herbal drugs have more than one marker substance, and individual analytical methods apply. Quantifying samples for different marker substances generates a huge analytical effort after the dissolution test is performed.

\section{Solubility Determination}

There are different approaches for the solubility determination of analytical markers. One possibility is to experimentally determine the thermodynamic $\mathrm{pH}$ dependent solubility using the shake-flask method as mentioned in USP <1092>, which is also a method for the evaluation of suitable dissolution media. Herein, the saturation solubility of the drug substance is determined in several buffer media over the physiological $\mathrm{pH}$ range. To ensure that saturation solubility is attained, a surplus of drug substance is used such that there is a balance between undissolved drug substance residue and drug substance in solution. In the case of herbal drugs, solubility of selected analytical markers in the matrix of the extract should be determined because these markers can have different physical and chemical properties, and thus different solubility. The presence of undissolved herbal drug residue does not correlate with reaching saturation solubility because other constituents may form the residue. Additionally, to quantify herbal markers dissolved in buffer media, herbal residues have to be tested for the presence of analytical markers to ensure saturation solubility is reached. Another approach to 
determine provisionally the $\mathrm{pH}$ dependent solubility of analytical herbal markers was reported by Lang et.al (12). The software program ADMET Predictor was used to estimate biopharmaceutically relevant descriptors such as solubility. Furthermore, the authors draw attention to extracts for which the active constituents are not known. Here, ADMET Predictor software was suggested as a useful tool for selecting marker substances based on their estimated solubility performance. In general, the significance of solubility tests for chemically defined herbal marker substances is limited for extracts for which constituents responsible for or contributing to the pharmacological activity are not known. The International Pharmaceutical Federation (FIP) Herbal Medicinal Products Working Group released a paper in 2003 concerning this (13). For extracts with known active constituents, in vitro characterization of the extract is proposed, while in vitro testing of extracts for which no marker substances are available is disputed due to uncertain correlation to in vivo performance. In such cases, the authors refer to in vivo studies instead of in vitro testing.

\section{Selection of Surfactant}

Severalanalyticalmarkersubstances of herbaldrugs exhibit lipophilic properties and thus low aqueous solubility. For dissolution testing of poorly soluble drugs, the addition of a surfactant may be necessary to enhance solubility. European and United States pharmacopeia suggest anionic surfactants such as sodium dodecyl sulfate (SDS), cationic surfactants such as cetyltrimethyl ammonium bromide (CTAB), as well as nonionic surfactants such as polysorbate 20 or polysorbate 80 . In contrast, Japanese Pharmacopoeia monographs on dissolution testing of poorly soluble drugs list only polysorbate 80 . For herbal drugs containing unknown constituents, a nonionic surfactant has the advantage of minimizing the risk of potential ion-to-ion interactions. A surfactant should exhibit a low critical micelle concentration (CMC) to reach sink conditions by the addition of small amounts in dissolution media. Thus, polysorbate 80 appears to be a suitable surfactant for dissolution testing of poorly soluble analytical marker substances of herbal drugs independent of regional regulations.

\section{REGULATIONS FOR DISSOLUTION TESTING OF HERBAL MEDICINAL PRODUCTS}

The regulatory classification of herbal medicinal products depends on regional legislation. Dissolution testing standards of herbal medicines exhibit regional differences as well as differences from regulations for chemical medicines. An overview of regulatory classification and dissolution standards for herbal medicinal products in Europe, the United States, and Asia is presented below. Table 1 summarizes the key points.

\section{Europe}

The manufacturing and quality of herbal medicinal products are regulated by EMA and harmonized throughout most of Europe. European Union monographs classify the regulatory status of herbal preparations into (1) well-established use, relying on sufficient safety and efficacy data in the literature, and (2) traditional use, based on long-term usage and experience. Traditional herbal products underlie a simplified registration procedure.

Efficacy of the constituents produces the same therapeutic effect in the isolated form as in the extract. In many cases, the herbal constituents responsible for the efficacy of the appropriate extract are not identified, or a synergy of multiple constituents is presumed. There is a distinction for constituents that are co-responsible for efficacy that do not produce the same effect in isolated form as in the extract, but show a contribution to the efficacy. To guarantee consistent batch-to-batch quality, the European Pharmacopoeia (EP) classifies herbal extracts into three categories, namely type A covering standardized extracts, type B1 related to quantified extracts, and type B2 linked to other extracts (Table 1). For standardized extracts that produce efficacy, the known pharmacologically active constituents serve as a quality attribute. If the efficacyproducing constituents are not known, a quantification of the native extract content is usual. Herein, analytical testing of marker substances serves as an additional quality attribute. This applies for both quantified extracts as well as for other extracts. For quantified extracts, marker substances are constituents known to contribute to pharmacological effects or have synergistic activity; they are co-responsible for efficacy. For other extracts for which constituents having pharmacological or clinical relevance are not known, a chemically defined constituent serves as a marker substance (11).

According to the EMA guideline on specifications (14), dissolution testing for immediate-release herbal medicinal products is required for those products containing standardized extracts. Analytical markers are used for determining dissolution profiles for constituents with known pharmacological activity. The permitted variability in the release rate is in accordance with regulations of chemical drugs, which is $10 \%$ of the labeled amount of herbal substance or herbal preparation. For immediaterelease herbal medicinal products containing quantified or other extracts, in vitro dissolution testing is not required. 
Table 1. Regulations for Dissolution Testing of Herbal Drugs in Europe, United States, Canada, and Asia

\begin{tabular}{|l|l|l|l|}
\hline Region & Regulatory Authority & Classification of Herbal Medicinal Products & Dissolution Testing \\
\hline Europe & EMA $^{a}$ & $\begin{array}{l}\text { Standardized extracts (Type A): Constituents with } \\
\text { pharmacological activity are known }\end{array}$ & $\begin{array}{l}\text { Required } \\
\text { EMA Guideline on specifications: test procedures } \\
\text { and acceptance criteria for herbal substances, } \\
\text { herbal preparations and herbal medicinal } \\
\text { products / traditional herbal medicinal products } \\
\text { (14) }\end{array}$ \\
\hline & & $\begin{array}{l}\text { Quantified extracts (Type B1): Constituents with } \\
\text { synergistic effect to pharmacological activity are } \\
\text { known }\end{array}$ & Not required \\
\hline & & $\begin{array}{l}\text { Other extract (Type B2): Constituents with } \\
\text { pharmacological activity or synergistic effect are } \\
\text { not known }\end{array}$ & Not required \\
\hline USA & Dietary Supplements: Botanical dosage forms & Required according to USP <2040> \\
\hline & FDA $^{b}$ & INDs (investigational new drug applications) & $\begin{array}{l}\text { Recommended according to FDA Guidance for } \\
\text { Industry: Botanical Drug Products }\end{array}$ \\
\hline Canada & & Natural Health Products & $\begin{array}{l}\text { Recommended according to Quality of Natural } \\
\text { Health Products Guide }\end{array}$ \\
\hline China & NNHPD & Traditional Chinese Medicine (TCM) / Natural & Not required \\
\hline Japan & Drugs & Not required \\
\hline South Korea & Kampo medicine & Not required \\
\hline & MHLW & Health functional food products & Not required \\
\hline & MFDS & Traditional Korean Medicine (TKM) / Hanbang & Not required \\
\hline
\end{tabular}

${ }^{a}$ European Medicines Agency

${ }^{b}$ Food and Drug Administration

${ }^{c}$ Natural and Non prescription Health Products Directorate

${ }^{d}$ China Food and Drug Administration

${ }^{e}$ Ministry of Health, Labor and Welfare

${ }^{f}$ Ministry for Food and Drug Safety

There is a concept paper (15) requesting a revision of the guideline on specifications; however, a change in the chapter covering dissolution testing is not scheduled. For controlled-release herbal medicinal products containing quantified or other extracts, analytical marker substances are selected for in vitro dissolution testing. These markers are herbal constituents showing little or no synergistic response to pharmacological activity, so correlation of in vitro dissolution profiles and in vivo performance is assumed.

\section{United States}

Herbal medicinal products are classified by the FDA as dietary supplements under the Dietary Supplement Health and Education Act (DSHEA) of 1994. Dietary supplements are classified into three categories: vitamin mineral dosage forms, botanical dosage forms, and other nutrients covering homeopathic and Ayuverdic remedies. The regulation defines dietary supplements as substances that supplement the diet but do not treat, diagnose, prevent, or cure diseases.
Dissolution testing of dietary supplements is regulated by USP $<2040>$. A six-fold determination of the dissolution profile of one or more analytical marker substances specified in the individual monograph is required. In addition to the testing of one botanical dosage unit per dissolution vessel, testing of two or more dosage units per vessel is permitted for botanical dosage forms. A release of at least $75 \%$ of the labeled herbal amount or herbal preparation within one hour is specified, unless the individual monograph does not regulate dissolution requirements.

In 2004, the FDA issued a guidance on botanical drug products (16) that states that approval of an herbal medicinal product is allowed as an investigational new drug application (IND) when proof of efficacy and safety is given. Due to the complex nature of herbal drugs and active constituents that are not well defined, FDA accepts reduced documentation of nonclinical safety and chemistry, manufacturing, and control (CMC) documents. Paragraphs for the setup of quality control specifications and studies for bioavailability and clinical pharmacology 
include recommendations for dissolution testing. A draft guidance on botanical drug development (17) was issued in 2015 without consideration for dissolution testing.

\section{Canada}

In Canada, any product with a therapeutic health claim is considered a drug according to the definition of the Food and Drugs Act (18). While products needing a prescription are regulated under the Food and Drugs Regulations, nonprescription products and natural products are regulated under the Natural Health Products Regulations (19), in which natural health products (NHP) are defined as vitamins and minerals, herbal remedies, homeopathic medicines, traditional medicines such as traditional Chinese medicines (TCM), as well as probiotics and other products such as amino acids and essential fatty acids. In this regard, the regulating authority for safety, effectiveness, and quality is the Natural and Non prescription Health Products Directorate (NNHPD) of Health Canada.

According to the guidance on natural health products (20), dissolution testing is required for NHPs of solid oral dosage forms. Especially for rapidly dissolving tablets and modified-release dosage forms including extended-release, combined-release, and timed-release tablets or capsules, dissolution testing is advised. For immediate-release and delayed-release dosage forms, either disintegration or dissolution testing should be performed. The guidance gives suggestions for dissolution measurement design, with single-point measurements for immediate-release dosage forms, multiple-point measurements for extended-release dosage forms, and two-point measurements using different media for delayed-release dosage forms.

The specification limits for dissolution test performance proposed in the guidance are complete dissolution within $45 \mathrm{~min}$ for uncoated tablets or capsules and within 60 min for caplets. Specification limits for modified-release dosage forms can be defined individually (e.g., on the basis of existing batch data). For extended-release products, data from in vitro-in vivo correlations can be used to set specification limits, if available. Specification limits for enteric-coated tablets are found in the USP or EP.

For the registration of herbal medicinal products in Canada, two guidance documents provide information regarding the kind and amount of data needed for license applications, one for NHP formulations based on traditional principles (21) and the other for any NHP that is not used as traditional medicine (22).

\section{China}

Herbal medicinal products in China are classified by the China Food and Drug Administration (CFDA) under the category Traditional Chinese Medicine (TCM)/Natural Drugs. TCM covers medical substances and preparations that apply to the guidance of Chinese traditional medical theory. In contrast, Natural Drugs are natural substances or preparations that apply to modern medical theory (23). Both TCM and Natural Drugs are considered as medicinal products, and thus regulatory requirements for TCM/ natural drugs are analogous to those for chemical drugs (24). However, application dossier requirements do not list dissolution testing as an explicit quality specification parameter for TCM and Natural Drugs. Furthermore, there is no regulation in the Chinese Pharmacopoeia for dissolution testing of TCM/Natural Drugs.

Registration of Western herbal medicinal drugs in China is also possible under the classification of medicinal product. There are nine different categories for approval listed in the CFDA guidance for Traditional Chinese Medicine and Natural Medicinal Products (25). Since 2010, CFDA has also accepted registration dossiers in accordance with ICH guidelines (23). Thus, for medicinal herbal products that are already registered in a country other than China, existing clinical, nonclinical, and technical data on quality and manufacturing are admitted by Chinese authorities. In addition to these data, in most cases CFDA requires local clinical testing for drug approval. For dissolution testing, the regulatory standards of the country of origin apply.

\section{Japan}

Japanese traditional herbal medicine, so-called Kampo medicine, arose from TCM of ancient China (26). With a long history of Kampo and its evolution over centuries, it is now an inherent part of the Japanese health care system. Kampo medicinal products are classified as pharmaceutical drugs along with chemical drugs. Monographs of Kampo drugs are included in the Japanese Pharmacopoeia (JP) and registered by the Ministry of Health, Labor, and Welfare (MHLW). Dissolution testing of Kampo medicinal products is not regulated by legislation.

Western herbal medicinal products that are already used outside Japan and are scientifically demonstrated to contain pharmacologically active constituents can be registered in Japan as pharmaceuticals according to the regulations of the National Institute of Health Sciences of Japan. Existing data of efficacy, dosage, and quality from clinical trials are admitted by Japanese authorities. However, drug safety has to be verified in Japanese 
patients by a clinical safety study. Regulatory standards for dissolution testing are guided by the country of origin standards.

\section{South Korea}

In South Korea, herbal medicinal products are classified by the Ministry for Food and Drug Safety (MFDS) into two categories depending on their claim, namely health functional food products and herbal medicine. The latter category is subdivided into products of Traditional Korean Medicine (TKM), which are also called Hanbang (27), and herbal medicinal products. Whereas TKM products undergo a simplified registration procedure according to TCM combination principles, herbal medicinal products need safety and efficacy evaluation for approval. Both categories are covered under the Korean health care system, National Health Insurance (NHI) (28), and are regulated by the MFDS of Korea. The quality of herbal medicinal products in Korea is regulated in the monograph Tests for Herbal Drugs of the Korean Pharmacopoeia as well as in appropriate individual monographs of the Korean Herbal Pharmacopoeia. For testing the quality of drugs, KFDA has issued a provision referring to specifications and test procedures (29). Dissolution testing is not part of the required quality specification parameters.

\section{CONCLUSION}

Dissolution testing is a well-established tool in pharmaceutical development and quality control for oral dosage forms. Several guidelines refer to the dissolution of chemical drugs. For herbal drugs, dissolution testing is also a helpful tool in pharmaceutical development and quality control, especially regarding batch-to-batch consistency. Dissolution testing for herbal drugs is much more complicated than for chemically defined drugs due to their complex nature. Because herbal medicinal products contain multiple active ingredients, the selection of analytical markers is critical. Additional considerations are necessary for solubility determination and selection of suitable dissolution media.

Regulatory standards for dissolution testing of herbal medicines exhibit regional differences. Furthermore, the regulations for herbal medicines are different from those for pharmaceutical medicines. The European Pharmacopoeia requires dissolution testing for immediate-release herbal products containing standardized extracts. In the United States, dissolution testing standards for botanical dosage forms are included in the USP. In China, Japan, and South Korea, the dissolution testing of herbal medicinal products is not regulated.

\section{REFERENCES}

1. Ekor, M. The growing use of herbal medicines: issues relating to adverse reactions and challenges in monitoring safety. Front. Pharmacol. 2014, 4, 177. DOI: 10.3389/fphar.2013.00177.

2. ICH guideline $Q 4 B$ annex 7 (R2) to note for evaluation and recommendation of pharmacopoeial texts for use in the $\mathrm{ICH}$ regions on dissolution test-general chapter; EMA/CHMP/ ICH/645469/2008; Committee for Medicinal Products for Human Use (CHMP), European Medicines Agency. London, 2010.

3. Siewert, M.; Dressman, J.; Brown, C. K.; Shah, V. P.; Aiache, J.M.; Aoyagi, N.; Bashaw, D.; Brown, W.; Burgess, D.; Crison, J.; et al. FIP/AAPS guidelines to dissolution/in vitro release testing of novel/special dosage forms. AAPS PharmSciTech 2003, 4 (1), 43-52. DOI: 10.1208/pt040107.

4. Noyes, A. A.; Whitney, W. R., The rate of solution of solid substances in their own solutions. J. Am. Chem. Soc. 1897, 19 (12), 930-934. DOI: 10.1021/ja02086a003.

5. Nelson, E. Solution Rate of Theophylline Salts and Effects from Oral Administration. J. Am. Pharm. Assoc. 1957, 46 (10), 607614. DOI: $10.1002 /$ jps.3030461012.

6. Waiver of In Vivo Bioavailability and Bioequivalence Studies for Immediate-Release Solid Oral Dosage Forms Based on a Biopharmaceutics Classification System; Guidance for Industry; U.S. Department of Health and Human Services, Food and Drug Administration, Center for Drug Evaluation and Research (CDER), U.S. Government Printing Office: Washington, DC, 2000.

7. Guideline on the Investigation of Bioequivalence; CPMP/EWP/ QWP/1401/98 Rev.1; Committee for Medicinal Products for Human Use (CHMP), European Medicines Agency: London, 2008.

8. Points to Consider on the Biopharmaceutical Characterization of Herbal Medicinal Products; EMEA/HMPWP/344/03; Working Party on Herbal Medicinal Products, The European Agency for the Evaluation of Medicinal Products: London, 2003.

9. General Guidelines for Methodologies on Research and Evaluation of Traditional Medicine, WHO/EDM/TRM/2000.1; World Health Organization: Geneva, 2000.

10. WHO guidelines for assessing quality of herbal medicines with reference to contaminants and residues; World Health Organization: Geneva, 2007.

11. Reflection paper on markers used for quantitative and qualitative analysis of herbal medicinal products and traditional herbal medicinal products; EMEA/HPMC/253629/2007 (Draft); Committee on Herbal Medicinal Products (HPMC), European Medicines Agency: London, 2008.

12. Waldmann, S.; Almukainzi, M.; Bou-Chacra, N. A.; Amidon, G. L.; Lee, B.-J.; Feng, J.; Kanfer, I.; Zuo, J. Z.; Wei, H.; Bolger, M. B.; Löbenberg, R. Provisional Biopharmaceutical Classification of Some Common Herbs Used in Western Medicine. Mol. Pharmaceutics 2012, 9 (4), 815-822. DOI: 10.1021/mp200162b. 
13. Lang, F.; Keller, K.; Ihrig, M.; Van Oudtshoorn-Eckard, J. Biopharmaceutical Characterization of Herbal Medicinal Products. Pharm. Forum 2003, 29 (4), 1337-1345.

14. Guideline on Specifications: Test Procedures and Acceptance Criteria for Herbal Substances, Herbal Preparations and Herbal Medicinal Products/Traditional Herbal Medicinal Products; EMA/ HPMC/162241/2005 Rev. 2; Committee for Medicinal Products for Human Use (CHMP), European Medicines Agency. London, 2011.

15. Concept paper on the revision of the guideline on specification: test procedures and acceptance criteria for herbal substances, herbal preparations and herbal medicinal products/traditional herbal medicinal products; EMA/HMPC/217753/2015; Committee on Herbal Medicinal Products (HPMC), European Medicines Agency: London, 2015.

16. Botanical Drug Products; Guidance for Industry; U.S. Department of Health and Human Services, Food and Drug Administration, Center for Drug Evaluation and Research (CDER), U. S. Government Printing Office: Washington, DC, 2004.

17. Botanical Drug Development; Guidance for Industry; U.S. Department of Health and Human Services, Food and Drug Administration, Center for Drug Evaluation and Research (CDER), U.S. Government Printing Office: Washington, DC, 2015.

18. Food and Drugs Act; Minister of Justice: Ottawa, Ontario, Canada, 2016.

19. Natural Health Products Regulations; Minister of Justice: Ottawa, Ontario, Canada, 2016.

20. Quality of Natural Health Products Guide; Natural and Nonprescription Health Products Directorate (NNHPD), Health Canada: Ottawa, Ontario, Canada, 2015.

21. Pathway for licensing Natural Health Products used as Traditional Medicines; Health Canada: Ottawa, Ontario, Canada, 2012.

22. Pathway for Licensing Natural Health Products Making Modern Health Claims; Health Canada: Ottawa, Ontario, Canada, 2012.

23. China Regulatory and Market Access Pharmaceutical Report; Pacific Bridge Medical: Bethesda, MD, 2014.

24. Wah, C. L.; Hock, S. C.; Yun, T. K., Current Scientific Status and Regulatory Control of Traditional/Herbal Medicinal Products: Globalization Challenges. Pharm. Eng. 2012, 32 (6), 10-20.

25. Notice on Approval for Clinical Trials of Import (including from Hong Kong, Macao and Taiwan) Traditional Chinese Medicine and Natural Medicinal Products; China Food and Drug Administration: Beijing, China, 2013.

26. Watanabe, K.; Matsuura, K.; Gao, P.; Hottenbacher, L.; Tokunaga, H.; Nishimura, K.; Imazu, Y.; Reissenweber, H.; Witt, C. M., Traditional Japanese Kampo Medicine: Clinical Research between Modernity and Traditional Medicine-The State of Research and Methodological Suggestions for the Future. Evidence-Based
Complementary Altern. Med. [Online] 2011, 2011, 513842. DOI: 10.1093/ecam/neq067.

27. Kumar, H.; Song, S.-Y.; More, S. V.; Kang, S.-M.; Kim, B.-W.; Kim, I.S.; Choi, D.-K. Traditional Korean East Asian Medicines and Herbal Formulations for Cognitive Impairment. Molecules 2013, 18 (12), 14670-14693. DOI: 10.3390/molecules181214670.

28. Lim, B. Korean medicine coverage in the National Health Insurance in Korea: present situation and critical issues. Integr. Med. Res. 2013, 2 (3), 81-88. DOI: 10.1016/j.imr.2013.06.004.

29. Provision for Specifications and Test Procedures of Drugs; Notification 2002-43; Department of Drug Evaluation, Korea Food and Drug Administration: Seoul, Korea, 2003. 